\title{
Novel Insights of Oligometastases and Oligo-Recurrence and Review of the Literature
}

\author{
Yuzuru Niibe ${ }^{1}$ and Joe Y. Chang ${ }^{2}$ \\ ${ }^{1}$ Department of Radiology and Radiation Oncology, School of Medicine, Kitasato University, 1-15-1 Kitasato, Minami-ku, \\ Kanagawa Sagamihara 252-0374, Japan \\ ${ }^{2}$ Department of Radiation Oncology, The University of Texas, MD Anderson Cancer Center, Houston, TX 77030, USA
}

Correspondence should be addressed to Yuzuru Niibe, joe-n@hkg.odn.ne.jp

Received 22 June 2012; Accepted 9 July 2012

Academic Editor: Hideomi Yamashita

Copyright (๑) 2012 Y. Niibe and J. Y. Chang. This is an open access article distributed under the Creative Commons Attribution License, which permits unrestricted use, distribution, and reproduction in any medium, provided the original work is properly cited.

\begin{abstract}
Oligometastases and oligo-recurrence are among the most important notions of metastatic and recurrent cancer. The concept of oligometastases is related to the notion that cancer patients with 1-5 metastatic or recurrent lesions that could be treated by local therapy achieve long-term survival or cure, while the concept of oligo-recurrence is related to the notion that cancer patients with 1-5 metastatic or recurrent lesions that could be treated by local therapy have controlled primary lesions. Achievement of long-term survival or cure in patients with oligometastases and oligo-recurrence is cancer and organ specific. These facts rely on the seed and soil theory and multiple steps of cancer progression. Oligo-recurrence is considered to have a better prognosis than oligometastases. In patients with oligometastases and oligo-recurrence, the oligometastases and oligo-recurrence are sometimes cured with only local therapy, which is an example of the abscopal effect, previously described in relation to cure of lesions outside of the field of radiation therapy without systemic therapy. Oligometastases and oligo-recurrence can now be cured by less invasive local treatment methods combined with systemic therapy. The mechanisms of oligometastases and oligo-recurrence, as well as novel insights into these important concepts, are presented in this paper.
\end{abstract}

\section{Introduction}

Oligometastases and oligo-recurrence are among the most important notions of metastatic and recurrent cancer $[1,2]$. These notions are now widely accepted by oncologists, and many reports of oligometastases and oligo-recurrence have been published. The concept of oligometastases is related to the notion that cancer patients with 1-5 metastatic or recurrent lesions that could be treated by local therapy achieve long-term survival or cure. However, the status of the primary lesion of these cancer patients has no restrictions, though patients with active primary lesions have a worse prognosis than patients with controlled primary lesions. Niibe et al. showed that the most important prognostic factor of oligometastases was the status of the primary lesion [3]. On the other hand, the concept of oligo-recurrence is related to the notion that cancer patients with 1-5 metastatic or recurrent lesions that could be treated by local therapy have controlled primary lesions [2]. Then, the biggest prognostic factor for oligometastases is overcome in oligo-recurrence. This is a very important point in oligo-recurrence. This notion has been proposed by Niibe et al. [2]. Another important point in oligo-recurrence is that the oligometastases are metachronous. Synchronous oligometastases have an active primary lesion. However, metachronous oligometastases almost always have a controlled primary lesion except for concomitant primary and distant recurrence (sites: 1-5).

Furthermore, achievement of long-term survival or cure in patients with oligometastases and oligo-recurrence is cancer- and organ-specific. These facts rely on the seed and soil theory and multiple steps of cancer progression $[4,5]$. The seed and soil theory remains an accepted notion in modern biology and oncology [6-8]. The cancer cells' interactions with host organs are very complicated and 
specific at the level of gene mutation, gene expression, molecular expression, MET-EMT cross-talk, and so on [610]. The multiple steps of cancer progression indicate that cancer cells in the primary lesion are not monoclonal and have a different metastatic potential [5]. Recently, cancer stem cells have been reported to play an important role in cancer progression and metastasis $[11,12]$.

In this paper, mechanisms of oligometastases and oligorecurrence are discussed through a review of the literature and our experience, and novel insights into these mechanisms are presented.

\section{Mechanisms of Oligometastases}

In this paper, oligometastases are defined as the state in which patients have 1-5 metastatic or recurrent lesions with active primary lesions. This definition prevents confusing oligometastases with oligo-recurrence. Another way of considering this status is sync-oligometastases, in which cancer patients have 1-5 synchronous metastases with active primary lesions, excluding metachronous metastases.

Metastasis has been recently reported to arise from cancer stem cells $[11,12]$. Primary tumor sites consist of various metastasis-potential cancer cells. Of these, cancer stem cells have metastasis potential, which is produced by cancer gene mutations. This means that sync-oligometastases cancer patients already have gene mutations in primary cancerous lesions $[5,11,12]$. Moreover, tumor-host cross-talk in gene mutations, gene expression, molecular expression, and METEMT interactions lead to organ-specific metastases $[5,6,9$, 10]. In nonsmall cell lung cancer (NSCLC), oligometastases often arise in patients who have brain-only or adrenal-only metastases $[3,13,14]$. In small cell lung cancer (SCLC), oligometastases often arise in patients who have brain-only metastases [15]. In uterine cervical cancer, oligometastases often arise in patients who have para-aortic lymph node-only metastases [16], while in colorectal cancer, oligometastases often manifest as liver-only metastases $[17,18]$. These syncoligometastases could be cured by local therapy combined with systemic therapy. In this situation, local therapy should treat both metastatic lesions and primary lesions to pursue cure or long-term survival.

Recently, Lussier et al. indicated that oligometastases enhanced by MicroRNA-200c lead to polymetastases after local radiation therapy [19]. This is a new finding related to cancer multistep progression. If MicroRNA-200c has not been enhanced in oligometastases, polymetastases do not occur. However, this has limitations, in that oligometastases occur in an organ-specific manner. This is explained by the above-mentioned modern seed and soil theory.

\section{Mechanisms of Oligo-Recurrence}

Oligo-recurrence is the state in which cancer patients have metachronous metastases after curative therapy for primary lesions. At recurrence, the cancer patients have no relapse of the primary lesions. This is very important with respect to local therapy. With local therapy it is relatively easy to treat 1-5 metastases and recurrences in one organ. However, primary lesion treatment is usually difficult with local therapy and includes radiation therapy, surgery, and radiofrequency ablative therapy, because primary lesion recurrence often involves regional lymph node metastases or invasion to adjacent organs. Furthermore, oligo-recurrence is the state of metachronous oligometastases. This is why we consider oligo-recurrence to have a better prognosis than sync-oligometastases.

Oligo-recurrence is also cancer and organ specific. The seed and soil theory is adapted in oligo-recurrence. In NSCLC, oligo-recurrence often arises with brain-only recurrences [3]. In uterine cervical cancer, oligo-recurrence often involves para-aortic lymph node-only recurrences [2022]. In colorectal cancer, oligo-recurrence often involves liver- and lung-only recurrences [17, 23].

At the time of treatment for the primary lesion, oligo-recurrent cancer patients might have one to several micrometastases. These micrometastases remain dormant for a period. These then grow and can be detected by computed tomography, magnetic resonance imaging, positron emission tomography, and increasing tumor marker levels. This state is oligo-recurrence, with one to several gross recurrences. Interleukin has been reported to play a key role in the growth of micrometastases [24]; it is the switch that results in progression of micrometastases.

\section{Relationship between the Abscopal Effect and Oligometastases and Oligo-Recurrence}

The abscopal effect is defined as tumor outside of the irradiation field disappearing without systemic therapy when the radiation therapy target tumor is irradiated. This is a rare phenomenon. We have reported the abscopal effect in uterine cervical cancer [25] and hepatocellular carcinoma [26]. Other reports have documented the abscopal effect in malignant melanoma [27], malignant lymphoma [28], and others.

In patients with oligometastases and oligo-recurrence, radiation oncologists, oncologic surgeons, and interventional oncologists have sometimes found that oligometastases and oligo-recurrence have been cured with only local therapy. These patients are considered to have micrometastases. However, gross metastases and recurrent lesions treated by radiation therapy, surgery, and radiofrequency ablative therapy lead to cure. This phenomenon is considered to be the abscopal effect. The abscopal effect is reported to occur with surgery, as well radiation therapy [29]. The abscopal effect could diminish micrometastases (Figure 1), so that oligometastases and oligo-recurrence treated only by local therapy may sometimes be cured.

\section{Relationship between Systemic Therapy and Oligometastases and Oligo-Recurrence}

Punglia et al. reported that the survival benefit of local therapy increased as systemic therapy improved [30]. Niibe et al. reported that the survival benefit of local therapy increased 


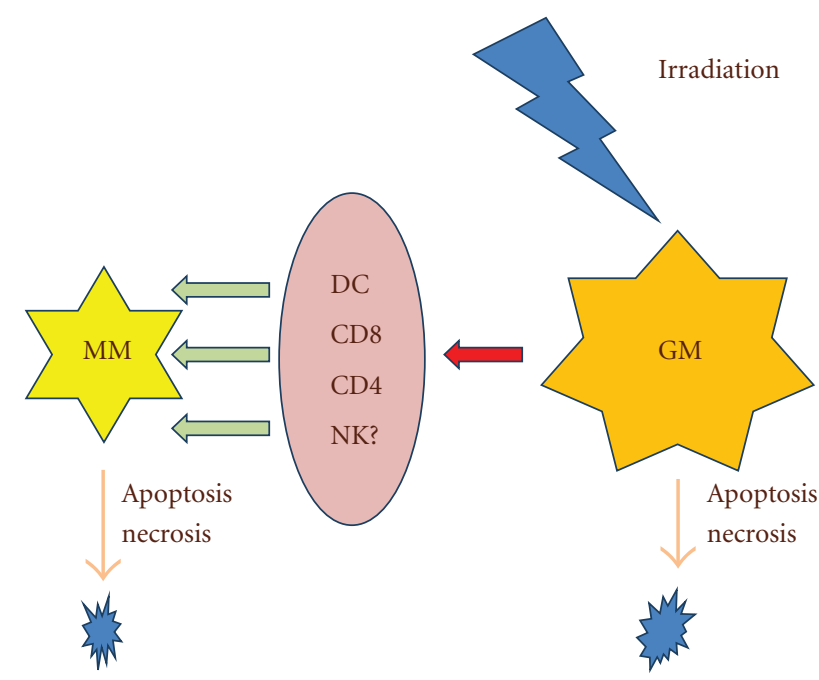

FIGURE 1: Relationship between the abscopal effect and disappearance of micrometastases. Abbreviations: GM: gross metastasis, DC: dendritic cell, NK: natural killer cell, MM: micrometastases.

dramatically as systemic therapy improved, indicating their original figure designating the sigmoid-curve relationship between increasing survival benefit of local therapy and improving systemic therapy [2]. This figure is very important because it revises the previous figure to a sigmoidcurve. Recently, systemic therapy has been improving, and the importance of local therapy, especially in cases with minimal invasiveness, is increasing dramatically. Stereotactic body radiation therapy (SBRT), intensity modulated radiation therapy (IMRT), proton therapy, heavy ion therapy, radiofrequency ablative therapy (RFA), video-assisted partial surgery, and robotic surgery are less invasive than therapies of a decade ago. These methods now apply to syncoligometastases and oligo-recurrence combined with systemic therapy, including molecular-targeted therapy. With these, patients can benefit from improved outcomes with less invasive and treatments that are more likely to be successful for sync-oligometastases and oligo-recurrence.

\section{Clinical Outcomes}

6.1. Oligometastases and Oligo-Recurrence in the Lungs. Oligometastases and oligo-recurrence in the lungs treated by surgery were reported to achieve good outcomes in the 1990s, in a large population study [31] (Table 1). The International Registry of Lung Metastases (IRLM) reported a 5 -year overall survival rate of $36 \%$ among 5206 patients with lung metastases treated by surgery. This report suggested that origin of the germ cell tumor was favorable survival. In 2009 , oligometastases of colorectal cancer in the lungs treated by surgery were also found to achieve favorable survival [32]. Three hundred and seventy-eight patients underwent pulmonary resection for colorectal cancer metastases with curative intent, and a 3-year overall survival rate of $78 \%$ was achieved. This indicated that oligometastases of colorectal cancer are favorable candidates for curative-intent therapy.
Since the 2000s, stereotactic body radiation therapy has rapidly spread as medical physics improved. Stereotactic body radiation therapy has been revealed to be equivalent to surgery in tumor ablation [33]. In oligometastases of the lungs, Okunieff et al. reported a local control rate of $94 \%$ (median follow-up: 18.7 months) and a 2-year progressionfree survival rate of $16 \%$ in patients treated with stereotactic body radiation therapy using mainly $50 \mathrm{~Gy} / 5 \mathrm{fr}$ [34]. Among them, cancer of breast or lung origin had better prognosis than those of other origins. Norihisa et al. reported a local control rate of $90 \%$ and an overall survival rate of $84.3 \%$ in patients after a 2-year followup using primarily 48-60 Gy/4$5 \mathrm{fr}$ [35], and suggested that no differences existed between different tumor origins. One prospective study also reported that the local control rate was $96 \%$ and overall survival rate was $39 \%$ in patients after a 2 -year followup [36]. In oligorecurrence of the lungs, Takahashi et al. reported a local control rate of $87 \%$ and an overall survival rate of $65 \%$ in patients after a 2 -year followup using $20-56 \mathrm{~Gy} / 1-7 \mathrm{fr}$, and suggested that those of colorectal cancer origin had a better prognosis than others [37]. Inoue et al. reported an overall survival rate of $54 \%$ in patients after a 5 -year followup using $40-48 \mathrm{~Gy} / 4 \mathrm{fr}$, and suggested that disease-free interval (DFI) $\geq 36$ was a significantly favorable prognostic factor [38].

6.2. Oligometastases and Oligo-Recurrence in the Liver. The most frequent liver metastases occur in colorectal cancer. However, colorectal cancer patients with liver metastases resected by surgery achieve favorable survival. The 5 -year overall survival rate is about $40 \%-50 \%[39,40]$ (Table 2). Furthermore, Adam et al. reported that initially unresectable colorectal liver metastasis could be cured by surgery after downsizing chemotherapy [41]. The cure rate was reported to be as high as $19 \%$. Bismuth also reported that initially unresectable colorectal liver metastases could achieve a 5year overall survival rate of $40 \%$ [42].

SBRT is also applied to liver metastases, as lung metastases can be curable by SBRT. van der Pool et al. reported that the 2-year local control and 2-year survival rates in colorectal cancer patients with liver metastases (i.e., mostly colorectal cancer) were $74 \%$ and $83 \%$, respectively, after treatment with SBRT using mainly $37.5 \mathrm{~Gy} / 3 \mathrm{fr}$ [43]. Romero et al. conducted a prospective trial to treat liver metastases with SBRT using mainly $37.5 \mathrm{~Gy} / 3$ fr [44], and reported that the 2 -year local control and 2-year overall survival rates were $86 \%$ and $62 \%$, respectively. Rusthoven et al. conducted a prospective trial on liver metastases treated with SBRT using 36-60 Gy/3 fr [45], and found that the 2-year local control and survival rates were $92 \%$ and $30 \%$, respectively, which indicated that favorable prognostic factors were the origins of colorectal cancer, breast cancer, and renal cell cancer.

\section{Conclusions}

The mechanisms of oligometastases and oligo-recurrence were reviewed, and novel insights are presented. Syncoligometastases and oligo-recurrence can now be cured by less invasive local treatment methods combined with systemic therapy. 
TABLE 1: Outcomes of oligometastases and oligo-recurrence in the lung.

\begin{tabular}{lccccc}
\hline Author & Year & Study design & Treatment method & Local control (\%) & Overall survival (\%) \\
\hline IRLM [31] & 1997 & retrospective & surgery & - & - \\
Onaitis et al. [32] & 2009 & retrospective & surgery & (5 years) \\
Okunieff et al. [34] & 2006 & retrospective & SBRT & 94 (median followup: 18.7 mo.) & 16 (PFS) \\
Norihisa et al. [35] & 2008 & retrospective & SBRT & 90 (2 years) & $84.3(2$ years) \\
Rusthoven et al. [36] & 2009 & prospective & SBRT & 96 (2 years) & $39(2$ years) \\
Takahashi et al. [37] & 2012 & retrospective & SRS, SBRT & 87 (2 years) & 65 (2 years) \\
Inoue et al. [38] & 2012 & retrospective & SBRT & - & $54(5$ years) \\
\hline
\end{tabular}

Abbreviations: SBRT: stereotactic body radiation therapy; SRS: stereotactic radiosurgery; mo.: months.

TABLE 2: Outcomes of oligometastases and oligo-recurrence in the liver.

\begin{tabular}{|c|c|c|c|c|c|}
\hline Author & Year & Study design & Treatment method & Local control (\%) & Overall survival (\%) \\
\hline Choti et al. [39] & 2002 & retrospective & surgery & - & 40 ( 5 years $)$ \\
\hline Pawlik et al. [40] & 2005 & retrospective & surgery & - & 58 (5 years) \\
\hline Adam et al. [41] & 2009 & retrospective & chemotherapy $\rightarrow$ surgery & - & 33 (5 years) \\
\hline Bismuth et al. [42] & 1996 & retrospective & chemotherapy $\rightarrow$ surgery & - & 40 (5 years) \\
\hline van der Pool et al. [43] & 2010 & retrospective & SBRT & 74 (2 years) & 83 (5 years) \\
\hline Romero et al. [44] & 2006 & prospective & SBRT & 86 (2 years) & 62 (5 years) \\
\hline Rusthoven et al. [45] & 2009 & prospective & SBRT & 92 (2 years) & 30 (5 years) \\
\hline
\end{tabular}

Abbreviations: SBRT: stereotactic body radiation therapy.

\section{References}

[1] S. Hellman and R. R. Weichselbaum, “Oligometastases," Journal of Clinical Oncology, vol. 13, no. 1, pp. 8-10, 1995.

[2] Y. Niibe and K. Hayakawa, "Oligometastases and oligorecurrence: the new era of cancer therapy," Japanese Journal of Clinical Oncology, vol. 40, no. 2, pp. 107-111, 2010.

[3] Y. Niibe, T. Nishimura, T. Inoue et al., "Oligometastases of brain only in patients with non-small cell lung cancer (NSCLC) treated with stereotactic irradiation (STI): a multiinstitutional study," International Journal of Radiation Oncology, vol. 78, no. 3, p. S497, 2010.

[4] S. Paget, "The distribution of secondary growths in cancer of the breast," The Lancet, vol. 133, no. 3421, pp. 571-573, 1889.

[5] A. F. Chambers, A. C. Groom, and I. C. MacDonald, "Dissemination and growth of cancer cells in metastatic sites," Nature Reviews Cancer, vol. 2, no. 8, pp. 563-572, 2002.

[6] I. J. Fidler, "The pathogenesis of cancer metastasis: the "seed and soil" hypothesis revisited," Nature Reviews Cancer, vol. 3, no. 6, pp. 453-458, 2003.

[7] D. Ribatti, G. Mangialardi, and A. Vacca, "Stephen Paget and the "seed and soil" theory of metastatic dissemination," Clinical and Experimental Medicine, vol. 6, no. 4, pp. 145-149, 2006.

[8] R. R. Langley and I. J. Fidler, "The seed and soil hypothesis revisited-the role of tumor-stroma interactions in metastasis to different organs," International Journal of Cancer, vol. 128, no. 11, pp. 2527-2535, 2011.

[9] T. Tsuji, S. Ibaragi, and G. F. Hu, "Epithelial-mesenchymal transition and cell cooperativity in metastasis," Cancer Research, vol. 69, no. 18, pp. 7135-7139, 2009.

[10] J. Monteiro and R. Fodde, "Cancer stemness and metastasis: therapeutic consequences and perspectives," European Journal of Cancer, vol. 46, no. 7, pp. 1198-1203, 2010.

[11] L. Vermeulen, F. de Sousa e Melo, D. J. Richel, and J. P. Medema, "The developing cancer stem-cell model: clinical challenges and opportunities," The Lancet Oncology, vol. 13, no. 2, pp. e83-e89, 2012.

[12] S. Badve and H. Nakshatri, "Breast-cancer stem cells-beyond semantics," The Lancet Oncology, vol. 13, no. 1, pp. e43-e48, 2012.

[13] J. L. Lopez Guerra, D. Gomez, Y. Zhuang et al., "Prognostic impact of radiation therapy to the primary tumor in patients with non-small cell lung cancer and oligometastasis at diagnosis," International Journal of Radiation Oncology, Biology, Physics. In press.

[14] D. J. Raz, M. Lanuti, H. C. Gaissert, C. D. Wright, D. J. Mathisen, and J. C. Wain, "Outcomes of patients with isolated adrenal metastasis from non-small cell lung carcinoma," Annals of Thoracic Surgery, vol. 92, no. 5, pp. 1788-1793, 2011.

[15] Y. Niibe, K. Karasawa, and K. Hayakawa, "Ten-year diseasefree survival of a small cell lung cancer patient with brain metastasis treated with chemoradiotherapy," Anticancer Research, vol. 24, no. 3, pp. 2097-2100, 2004.

[16] Y. Niibe, T. Nakano, T. Ohno, Y. Suzuki, K. Oka, and H. Tsujii, "Prognostic significance of c-erbB-2/HER2 expression in advanced uterine cervical carcinoma with para-aortic lymph node metastasis treated with radiation therapy," International Journal of Gynecological Cancer, vol. 13, no. 6, pp. 849-855, 2003.

[17] D. J. Gallagher and N. Kemeny, "Metastatic colorectal cancer: from improved survival to potential cure," Oncology, vol. 78, no. 3-4, pp. 237-248, 2010.

[18] N. Mahmoud and K. B. Dunn, "Metastasectomy for stage IV colorectal cancer," Diseases of the Colon and Rectum, vol. 53, no. 7, pp. 1080-1092, 2010.

[19] Y. A. Lussier, H. R. Xing, J. K. Salama et al., "Microrna expression characterizes Oligometastasis(es)," PLoS One, vol. 6, no. 12, Article ID e28650, 2011.

[20] Y. Niibe, M. Kenjo, T. Kazumoto et al., "Multi-institutional study of radiation therapy for isolated para-aortic lymph 
node recurrence in uterine cervical carcinoma: 84 subjects of a population of more than 5,000," International Journal of Radiation Oncology Biology Physics, vol. 66, no. 5, pp. 13661369, 2006.

[21] Y. Niibe, T. Kazumoto, T. Toita et al., "Frequency and characteristics of isolated para-aortic lymph node recurrence in patients with uterine cervical carcinoma in Japan: a multiinstitutional study," Gynecologic Oncology, vol. 103, no. 2, pp. 435-438, 2006.

[22] E. Y. Huang, Y. J. Huang, C. C. Chanchien et al., "Pretreatment carcinoembryonic antigen level is a risk factor for paraaortic lymph node recurrence in addition to squamous cell carcinoma antigen following definitive concurrent chemoradiotherapy for squamous cell carcinoma of the uterine cervix," Radiation Oncology, vol. 7, no. 1, article 13, 2012.

[23] S. Limmer and L. Unger, "Optimal management of pulmonary metastases from colorectal cancer," Expert Review of Anticancer Therapy, vol. 11, no. 10, pp. 1567-1575, 2011.

[24] G. P. Dunn, C. M. Koebel, and R. D. Schreiber, "Interferons, immunity and cancer immunoediting," Nature Reviews Immunology, vol. 6, no. 11, pp. 836-848, 2006.

[25] M. Takaya, Y. Niibe, S. Tsunoda et al., "Abscopal effect of radiation on toruliform para-aortic lymph node metastases of advanced uterine cervical carcinoma-a case report," Anticancer Research, vol. 27, no. 1, pp. 499-503, 2007.

[26] K. Okuma, H. Yamashita, Y. Niibe, K. Hayakawa, and K. Nakagawa, "Abscopal effect of radiation on lung metastases of hepatocellular carcinoma: a case report," Journal of Medical Case Reports, vol. 19, no. 5, article 111, 2011.

[27] M. A. Postow, M. K. Callahan, C. A. Barker et al., "Immunologic correlates of the abscopal effect in a patient with melanoma," New England Journal of Medicine, vol. 366, no. 10, pp. 925-931, 2012.

[28] M. P. Nobler, "The abscopal effect in malignant lymphoma and its relationship to lymphocyte circulation," Radiology, vol. 93, no. 2, pp. 410-412, 1969.

[29] D. Perego and A. Faravelli, "Unexpected consequence of splenectomy in composite lymphoma. The abscopal effect," Haematologica, vol. 85, no. 2, p. 211, 2000.

[30] R. S. Punglia, M. Morrow, E. P. Winer, and J. R. Harris, "Local therapy and survival in breast cancer," New England Journal of Medicine, vol. 356, no. 23, pp. 2399-2405, 2007.

[31] U. Pastorino, M. Buyse, G. Friedel et al., "Long-term results of lung metastasectomy: prognostic analyses based on 5206 cases," Journal of Thoracic and Cardiovascular Surgery, vol. 113, no. 1, pp. 37-49, 1997.

[32] M. W. Onaitis, R. P. Petersen, J. C. Haney et al., "Prognostic factors for recurrence after pulmonary resection of colorectal cancer metastases," Annals of Thoracic Surgery, vol. 87, no. 6, pp. 1684-1688, 2009.

[33] H. Onishi, H. Shirato, Y. Nagata et al., "Stereotactic body radiotherapy (SBRT) for operable stage I non-small-cell lung cancer: can SBRT be comparable to surgery?" International Journal of Radiation Oncology, Biology, Physics, vol. 81, no. 5, pp. 1325-1358, 2011.

[34] P. Okunieff, A. L. Petersen, A. Philip et al., "Stereotactic Body Radiation Therapy (SBRT) for lung metastases," Acta Oncologica, vol. 45, no. 7, pp. 808-817, 2006.

[35] Y. Norihisa, Y. Nagata, K. Takayama et al., "Stereotactic body radiotherapy for oligometastatic lung tumors," International Journal of Radiation Oncology Biology Physics, vol. 72, no. 2, pp. 398-403, 2008.

[36] K. E. Rusthoven, B. D. Kavanagh, S. H. Burri et al., "Multiinstitutional phase I/II trial of stereotactic body radiation therapy for lung metastases," Journal of Clinical Oncology, vol. 27, no. 10, pp. 1579-1584, 2009.

[37] W. Takahashi, H. Yamashita, Y. Niibe, K. Shiraishi, K. Hayakawa, and K. Nakagawa, "Stereotactic body radiotherapy for metastatic lung cancer as oligo-recurrence: an analysis of 42 cases," Pulmonary Medicine. In press.

[38] T. Inoue, N. Katoh, R. Onimaru, and H. Shirato, "Clinical outcomes of stereotactic body radiotherapy for patients with lung tumors in the state of oligo-recurrence," Pulmonary Medicine, vol. 2012, Article ID 369820, 5 pages, 2012.

[39] M. A. Choti, J. V. Sitzmann, M. F. Tiburi et al., "Trends in longterm survival following liver resection for hepatic colorectal metastases," Annals of Surgery, vol. 235, no. 6, pp. 759-766, 2002.

[40] T. M. Pawlik, C. R. Scoggins, D. Zorzi et al., "Effect of surgical margin status on survival and site of recurrence after hepatic resection for colorectal metastases," Annals of Surgery, vol. 241, no. 5, pp. 715-724, 2005.

[41] R. Adam, D. A. Wicherts, R. J. Haas et al., "Patients with initially unresectable colorectal liver metastases: is there a possibility of cure?" Journal of Clinical Oncology, vol. 27, no. 11, pp. 1829-1835, 2009.

[42] H. Bismuth, R. Adam, F. Lévi et al., "Resection of nonresectable liver metastases from colorectal cancer after neoadjuvant chemotherapy," Annals of Surgery, vol. 224, no. 4, pp. 509-522, 1996.

[43] A. E. M. van der Pool, A. M. Romero, W. Wunderink et al., "Stereotactic body radiation therapy for colorectal liver metastases," British Journal of Surgery, vol. 97, no. 3, pp. 377$382,2010$.

[44] A. M. Romero, W. Wunderink, S. M. Hussain et al., "Stereotactic body radiation therapy for primary and metastatic liver tumors: a single institution phase i-ii study," Acta Oncologica, vol. 45, no. 7, pp. 831-837, 2006.

[45] K. E. Rusthoven, B. D. Kavanagh, H. Cardenes et al., "Multiinstitutional phase I/II trial of stereotactic body radiation therapy for liver metastases," Journal of Clinical Oncology, vol. 27, no. 10, pp. 1572-1578, 2009. 


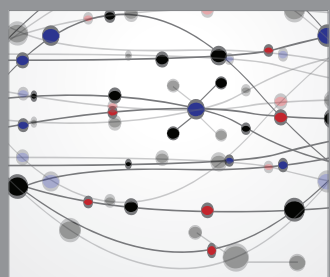

The Scientific World Journal
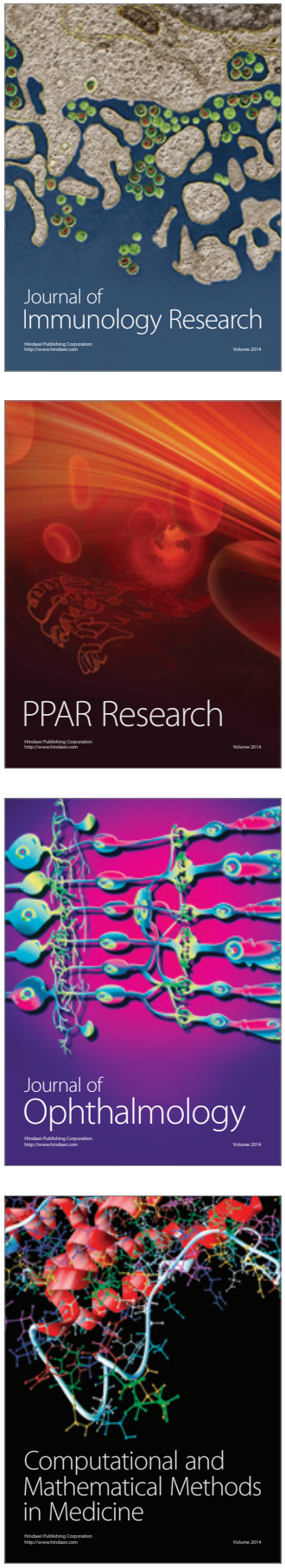

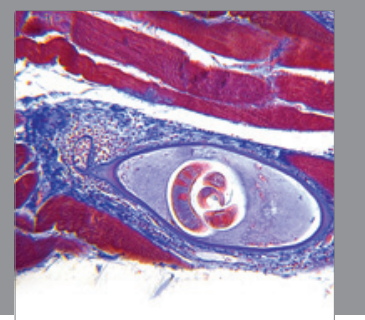

Gastroenterology

Research and Practice
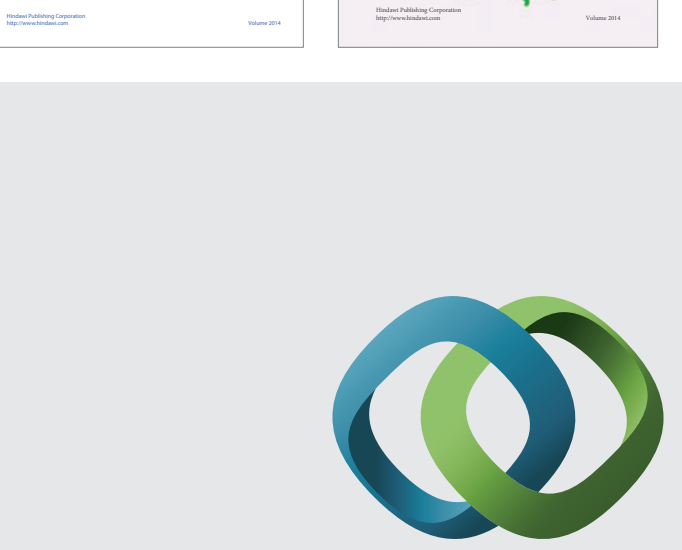

\section{Hindawi}

Submit your manuscripts at

http://www.hindawi.com
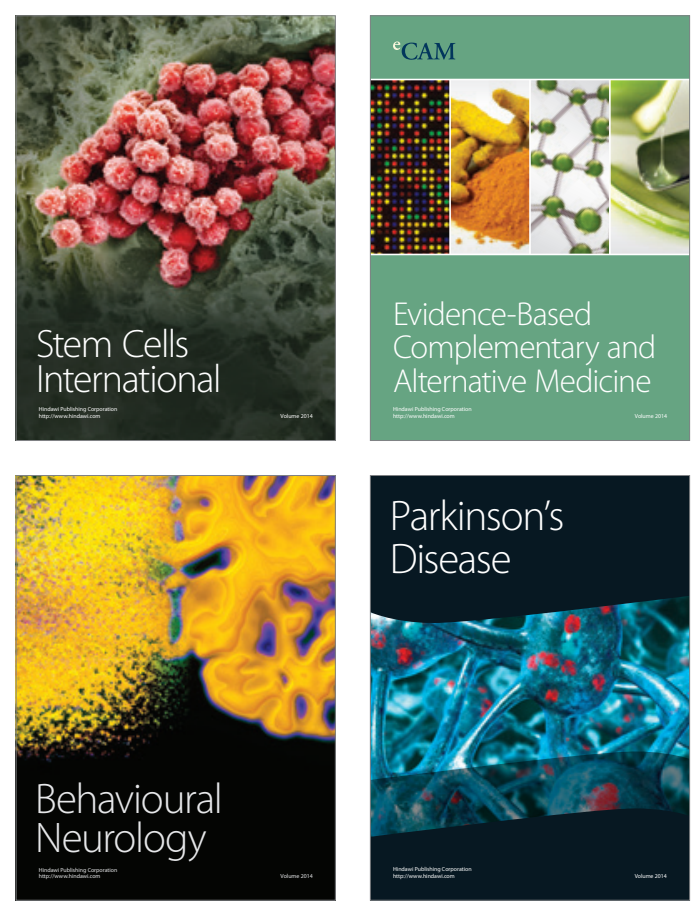

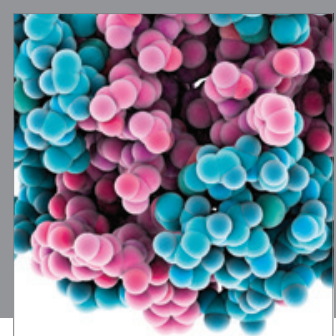

Journal of
Diabetes Research

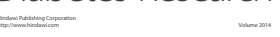

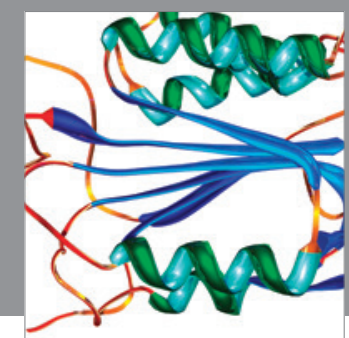

Disease Markers
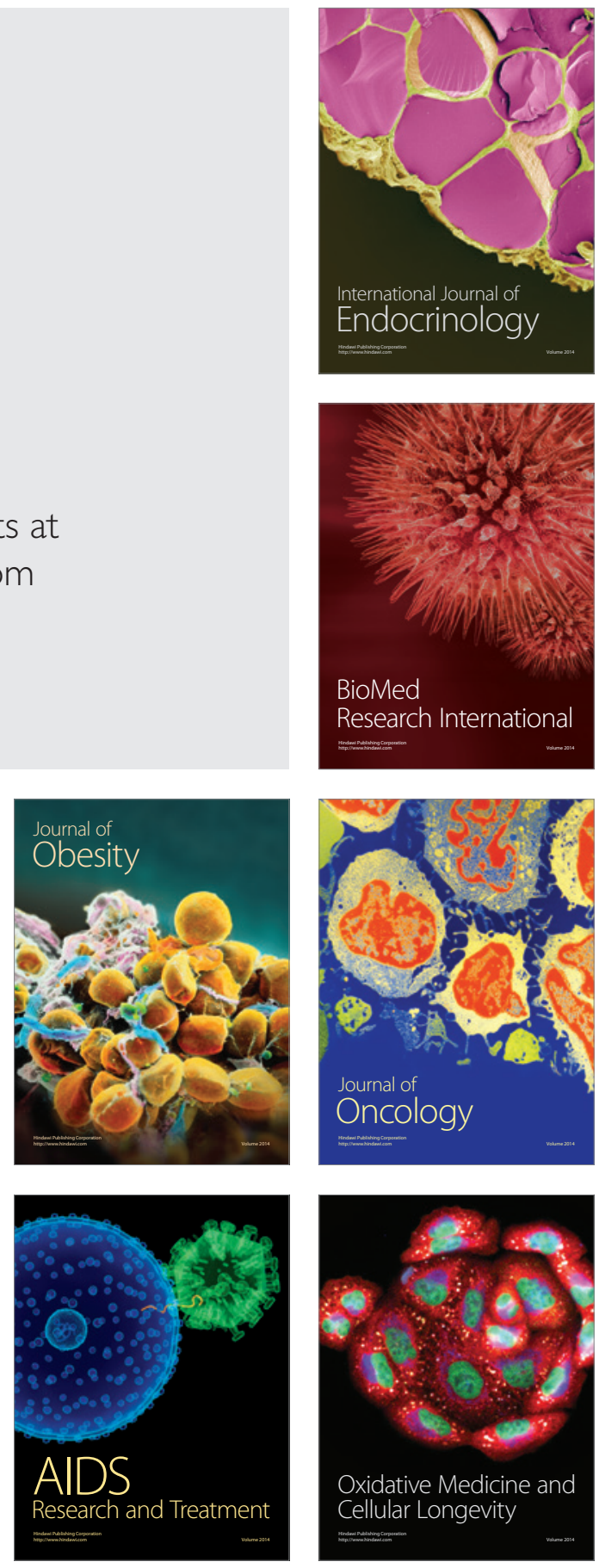